\title{
An approach to reliability assessment of complex systems at CERN
}

\author{
Miriam Blumenschein, MSc., CERN \\ Jelena Spasic, PhD., CERN \\ Jens Steckert, MSc., CERN \\ Jan Uythoven, PhD., CERN
}

Key Words: Reliability requirements, risk evaluation, context dependent functions, risk matrix

\section{SUMMARY \& CONCLUSIONS}

This paper presents the systematic approach Reliability Requirements and Initial Risk Evaluation (RIRE) developed and used at CERN. RIRE is a four-step procedure, which provides a framework for the experience based derivation of quantitative reliability targets for CERN's accelerator systems. These targets are not subject to statutory regulations. RIRE shows the risks posed by a system and prioritizes subsequent, more detailed analyses, such as Fault Tree. The application of RIRE to the quench detection system of the LHC super conducting magnets is shown in this paper. From this example it is concluded that RIRE is suitable for the analysis of a complex system with context dependent functions.

\section{INTRODUCTION}

The European Organization for Nuclear Research CERN is the largest particle physics laboratory in the world. With particle accelerators, detectors and computing facilities, nature's basic constituents are studied to improve our understanding of the universe. With the construction of the most complex scientific instrument on earth, the Large Hadron Collider LHC (Figure 1), reliability and availability of the accelerator became additional performance indicators [1].

The LHC, which started operation in 2008, presently accelerates two counter-rotating proton beams to a maximum collision energy of $13 \mathrm{TeV}$ in a $27 \mathrm{~km}$ circumference tunnel [3]. Each particle beam has a stored energy of 362 MJ. This corresponds to the energy content of one $200 \mathrm{~m}$ long train at the speed of around $150 \mathrm{~km} / \mathrm{h}$. Around 10000 superconducting magnets steer the particle beams in the LHC. Among them, $1232,14.3 \mathrm{~m}$ long main dipole magnets provide a nominal field of $8.3 \mathrm{~T}$ to deflect the beams and $392,3.25 \mathrm{~m}$ long main quadrupole magnets provide a $223 \mathrm{~T} / \mathrm{m}$ gradient field to focus the particle beams and keep them near the reference orbit. The required magnetic fields are achieved using superconducting technology. The energy stored in the superconducting magnet circuits exceeds $10 \mathrm{GJ}$, the energy content of about $230 \mathrm{~kg}$ gasoline.

Due to the large amount of energy stored in the beams and in the magnet systems, operation with high intensity beams is challenging and bears risks of equipment damage [4].
Uncontrolled release of energy can cause major damage of the accelerator equipment, which would cause long stops for repair. As an example, the exchange of a superconducting magnet requires around three months. Safe operation of the LHC relies therefore on a sophisticated machine protection system.

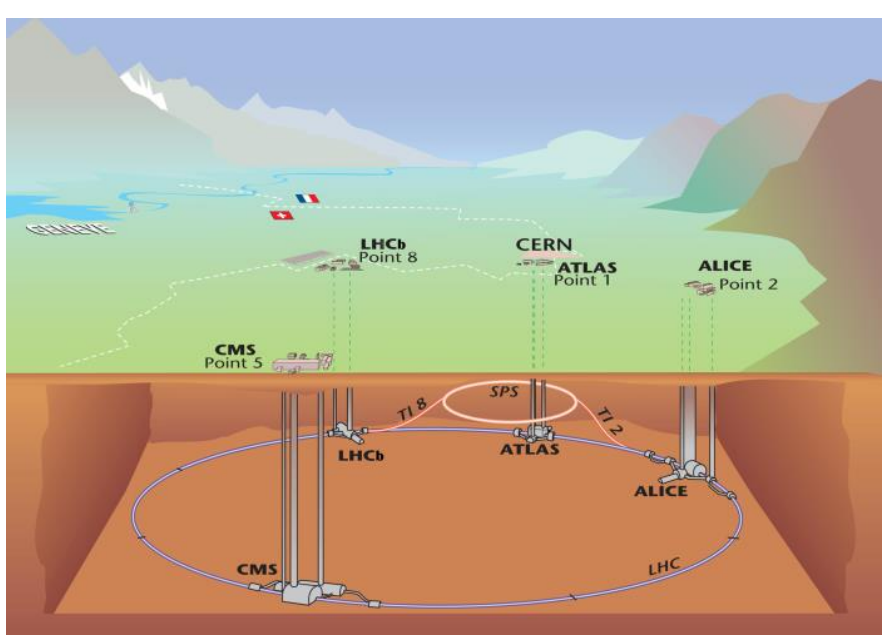

Figure 1: The Large Hadron Collider LHC [2]

A part of the machine protection subsystem, which protects the superconducting magnets, is the quench detection system [4]. The superconducting LHC magnets operate at $1.9 \mathrm{~K}$. If, due to an impact of the beam or a mechanical disturbance, the critical temperature of the superconductor in the magnet coils is exceeded, the superconducting state disappears. The transition from the superconducting to the resistive conducting state is called a quench. When a quench occurs, unless precautions are taken, the temperature in the resistive zone would increase to $1000 \mathrm{~K}$ within less than a second, destroying the magnet. Since quenches are unavoidable, a reliable quench detection system is required.

Reliability has been considered as a crucial goal for the design of the machine protection system and its subsystems like the quench protection system [3]. No legal requirements concerning facility operation or product liability, as for example the machinery directive [5], apply to CERN's machine protection system. Legal requirements only apply to the personal protection system at CERN, which is not considered 
as a part of the machine protection system. The strict application of industrial safety standards, as for example IEC 61508 [6], is therefore not required for the machine protection system. Instead, well-established methodologies and tools like risk matrix [7], failure mode and effect analysis (FMEA) [8] and fault tree analysis [9] are combined with best practice at CERN. The result is a method for reliability requirements and initial risk evaluation for CERN's systems, which are not subject to statutory regulations.

\section{PRINCIPLE OF RIRE}

Reliability Requirements and Initial Risk Evaluation RIRE (French for laugh) is a systematic procedure for the first step of a reliability analysis applied to CERN's accelerator systems, which are not subject to statutory regulations.

As proposed by design methodologies [10], designs are based on a functional specification document, which determines what is to be built, and for which purpose, without giving technical solutions or details. Reliability requirements are typically part of the specification. The objective is the definition and control of system performance on a high and general level.

The underlying principle of RIRE (Figure 2) is that the accelerator reliability targets - the overall accelerator reliability needs - are allocated, via the systems' failure effects, to the systems' failure modes. Failure modes and effects are derived from failure analysis with FMEA. Attributes of the system failure modes are used to estimate the dimension of the necessary reliability actions.

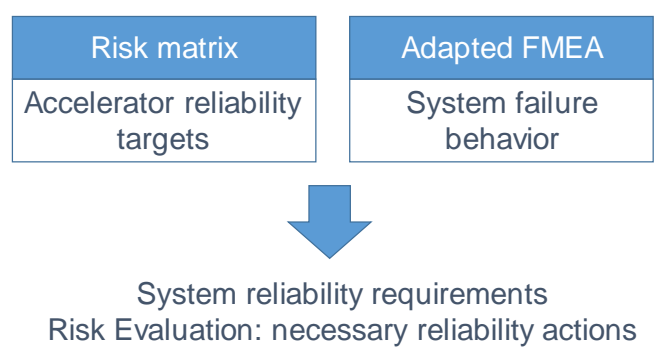

Figure 2: Principle of RIRE

\section{DETAILED STEPS OF RIRE}

The RIRE procedure consists of four steps. Two preparatory steps provide the foundation. First, the accelerator reliability requirements are defined with a risk matrix. Then, the definition of system failures, the estimation of its severity and other attributes are achieved with an adapted FMEA. Finally, two subsequent steps deliver the results, the system reliability targets, and risk evaluation and prioritization. In the following sections the RIRE procedure is presented stepwise, using the example of the quench detection system of the main quadrupole magnets of the LHC.

\subsection{Step 1: Accelerator Risk Matrix}

In [11] risk is defined as "the probability of occurrence of harm and the severity of that harm". A risk matrix [7] graphically illustrates risk with the severity on one axis and the probability of its occurrence (frequency) on the other. The content of a cell indicates the estimated risk level for the specific combination of severity and probability. The risk level can be a qualitative expression or an index, e.g. from one to six. Risk matrices are a common tool in risk management and they exist in numerous versions, as for example ALARP [12].

For the LHC, the risk matrix is used to define the accelerator reliability requirements, see Figure 3 . The severity, an estimate of how strongly the system failure will affect the operation of the LHC, is described with the recovery time criterion. Recovery time includes the time needed for maintenance or intervention and the time to bring the LHC back to the state at which the failure occurred. Seven severity classes and eight frequency classes are distinguished. The risk level is binary, that is acceptable risk cells are green and unacceptable ones are red. The acceptable LHC risk is defined for each severity class based on experience. For example, recovery time of the order of hours is acceptable once per week, accounting for all accelerator systems. A recovery time of a year is assumed to be acceptable once per 100 years, so unlikely in the lifetime of the accelerator but not excluded.

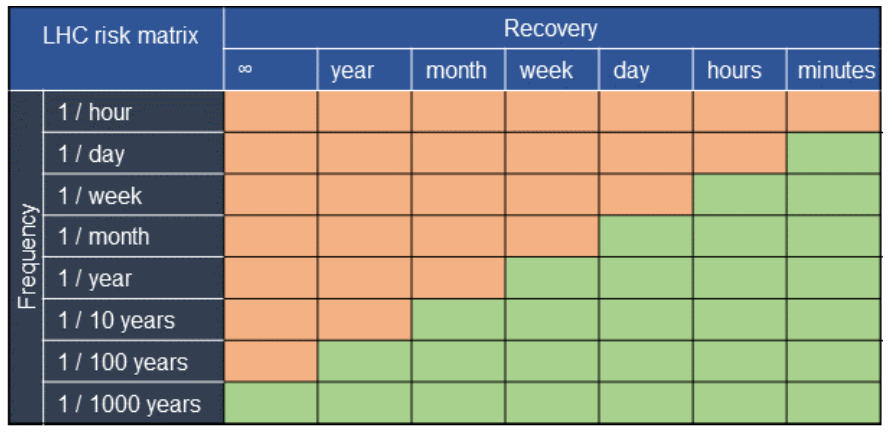

Figure 3: LHC risk matrix

\subsection{Step 2: RIRE tailored FMEA}

For the system under study, the failure modes and effects have to be identified and allocated to the accelerator reliability requirements. To determine the priority of further studies, relevant characteristics, like severity and detectability have to be identified. These actions are classically achieved through the inductive methodology Failure Modes and Effects Analysis FMEA, as described in [8]. Being traditionally a flexible tool, FMEA is applied in diverse sectors, as for example engineering, medical sector or education. While the principle of the methodology remains the same, there are wide variations in the manner in which the FMEA is performed and presented. In the following subsections, the FMEA tailored to meet the specific RIRE needs is introduced.

\subsubsection{System Context}

First, a block diagram of the system under evaluation is constructed. The purpose of the block diagram is to demonstrate the system's functional position and importance. It shows the system itself and all those neighboring systems it interfaces to, to be able to define and understand the system functionality. 
Figure 4 shows the block diagram of the quench detection system for the LHC main quadrupoles for one out of eight LHC sectors. The superconducting quadrupoles are powered in two circuits, each time 51 quadrupole are connected in series. The quench detection system surveys the voltage over each individual magnet. If it detects a quench, it activates three protection mechanisms. By opening the quench interlock loop, the energy extraction system is activated and a beam dump is triggered. The beam dump is the emergency stop of the accelerator during which the particle beams are extracted from the accelerator and directed to an external dump block, where the energy is safely dissipated. The energy extraction system, as a second protection system, dissipates the energy stored in the magnet circuit into the dump resistor. The third protection measure are quench heaters which are energized by the quench detection system. They spread the heat over the entire magnet to distribute the energy. These three active protection measures all rely on the correct functioning of the quench detection system.

The block diagram illustrated in Figure 4 is simplified. For the success of the methodology, it is important to be specific and to use the real names and abbreviations.

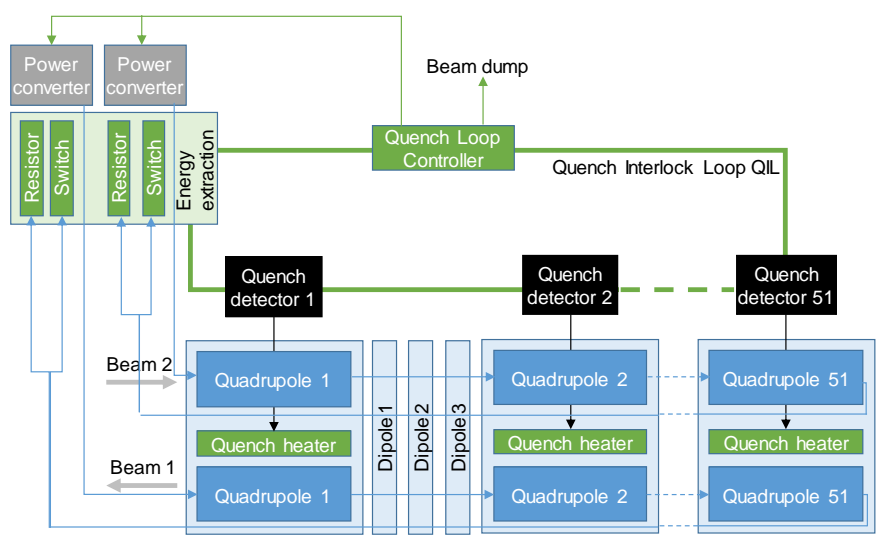

Figure 4: Simplified block diagram of the LHC quench detection system

\subsubsection{System Structure}

Ordering the elements of the block diagram hierarchically is the basis for determining the system functions, failure modes and failure effects. The hierarchical decomposition is displayed as a structure tree (Figure 5).

\section{Quadrupole

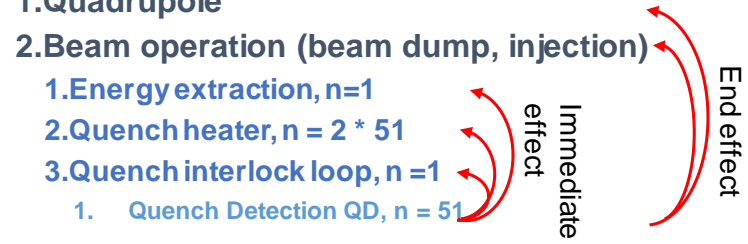

\section{Figure 5: Structure tree}

The highest level corresponds to the accelerator, for which failure effects are defined, while the lowest one corresponds to the system, for which failures modes are defined. Intermediate levels can be added for the purpose of clarification.

The quench detection system is the lowest indenture level. For clarity, the neighboring systems, energy extraction, quench heater and quench interlock loop, are added as intermediate level. The highest level is the quadrupole and beam operation.

\subsubsection{System Functions}

To determine the system functions within a larger system, a black box approach is used. The system is shown as a black box, neglecting the inner working and putting the focus on the inputs and their origin as well as the outputs and their destination. The connection between inputs and outputs are the system functions. Categories of inputs and outputs include flow of information, flow of energy and flow of the end product. In contrast to the block diagram, it is important to include all redundancies.

Two voltage taps per quadrupole provide the quench detection system with information (Figure 6). In case of a quench, energy is discharged into two quench heater $(\mathrm{QH})$ circuits. The manipulation of the quench interlock loop is redundant. The quench detection system provides logging data, out of which the heater and quench detection status is derived. Post mortem (PM) data is sent after a quench event. Via the supervision tool, experts can interact.

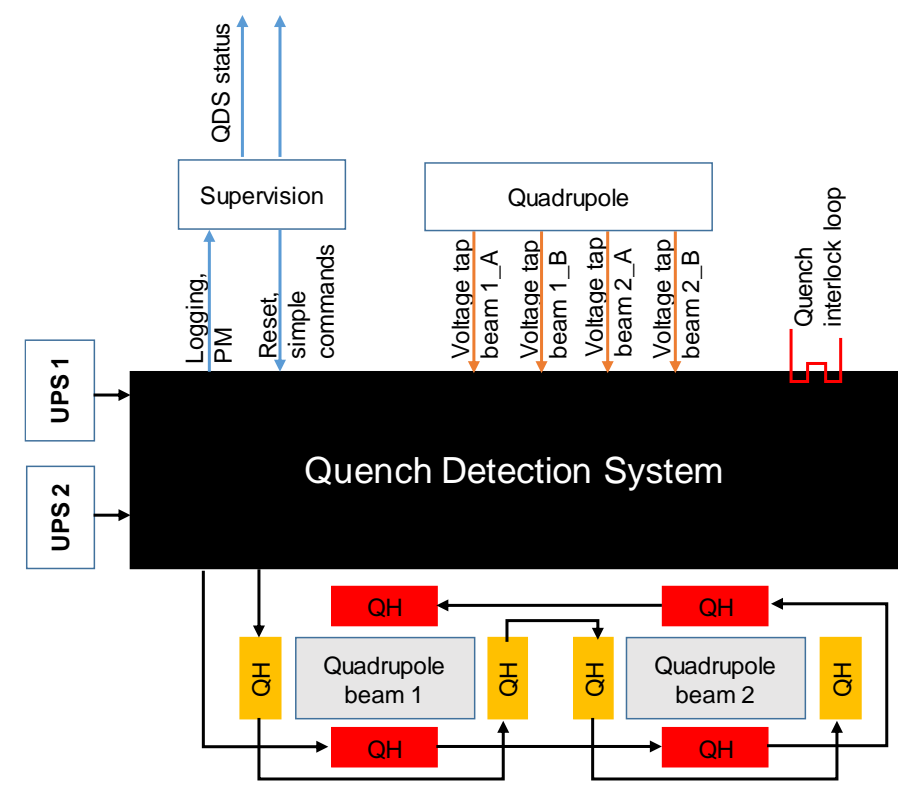

Figure 6: Quench detection system as a black box

\subsubsection{Context Dependent Functions}

The function of the system is the generation of the outputs of the black box. Each output corresponds to one function and is formulated in the form "verb subject" (e.g. provide quench heater current). The system might operate in different contexts - the state of the system or of the environment - and the functions might change with and depend on the context, e.g., provide quench heater current when quench appears.

This dynamic system behavior is described with a state diagram, a tool commonly used in computer science [13]. The 
state diagram shows the possible system states and the initiators for the transitions, the progression from one state to another. Initiators are internal or external events.

Figure 7 shows the state diagram of the quench detection system. Rounded boxes represent the states, the color code indicates the environment variables and arrows represent the transitions.

In the state "normal operation", the quadrupole circuit is energized and the LHC is, from the quench detection system point of view, ready for operation. Depending on the LHC operational state, the particle beam is present or not. If a magnet quenches, the quench detection system fulfils its protection function and moves to the state "post quench I", where post mortem data is collected. Sending of the post mortem data moves the system to the state "quench event analysis", where the quench event is analyzed by experts. If malfunctioning is revealed, the system moves to a maintenance state. If not, the quench detection system is prepared for another operational cycle by resetting the detection board. Once the capacitor bank is charged, the quench detection system is ready for operation and moves back to the state "normal operation".

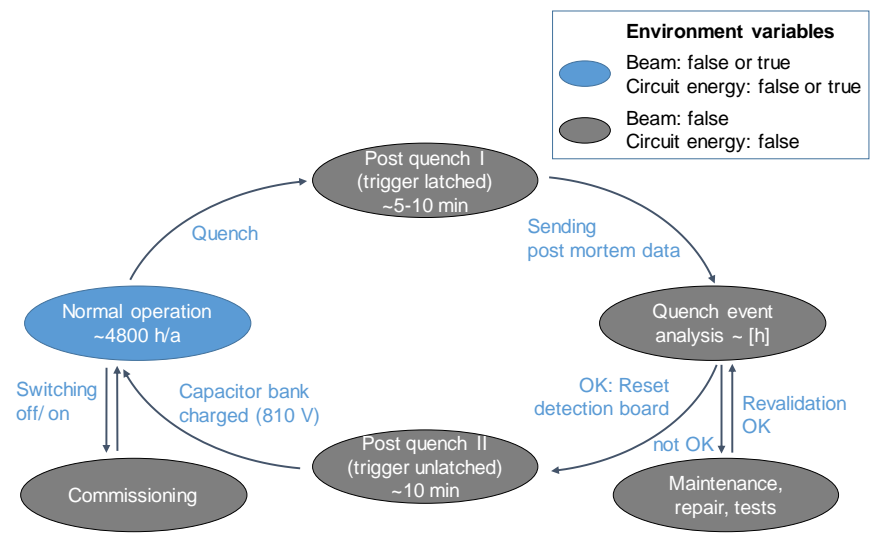

Figure 7: State diagram for the quench detection system

The context dependent functions are obtained by adapting the black box functions to each state and transition. Figure 8 shows the quench detection functions for two states, "normal operation" and "post quench I", and one transition, "quench".

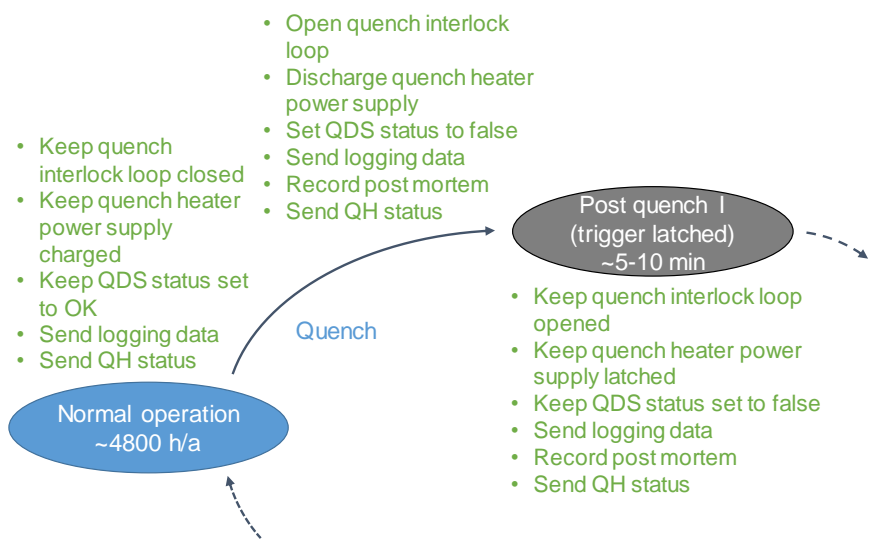

Figure 8: Context dependent functions
The functions for the output "quench interlock loop" (Figure 6) are for example: keep quench interlock loop closed (state normal operation), open quench interlock loop (transition quench) and keep quench interlock loop opened (state post quench I).

With the state diagram, not only equipment functions are identified, but also control actions of operators. For the quench detection system this is especially the case for the state "quench event analysis".

\subsubsection{Failure Modes and Effects}

The comprehensive determination of the context dependent functions ensures the completeness of failure modes and effects. This is crucial, since reliability characteristics are assigned to each failure effect. The completeness of failure modes is important for further analyses.

The FMEA table tailored to RIRE is illustrated in Table 1. The content of the first two rows in the FMEA table is obtained with the state diagram and the context dependent functions. The failure modes are the negation of the context dependent functions. The row immediate effect has descriptive character with the purpose of clarification and is optional. Failure effects are on the indenture level of the accelerator. Their definition is important since reliability requirements are defined in the accelerator level. For each end effect the severity category is estimated based on the recovery time (Table 2). The recovery time corresponds to the risk matrix columns. The detection method refers to the detectability of the occurred failure mode. The row recommendations proposes modifications to the design or to testing procedures.

\section{Table 1: Excerpt of the FMEA table}

\begin{tabular}{|c|c|c|c|}
\hline \multicolumn{4}{|c|}{ FMEA black box level: quench detection system } \\
\hline Context & $\begin{array}{l}\text { Normal operation of } \\
\text { QPS }(\sim 4800 \mathrm{~h} / \mathrm{a})\end{array}$ & $\begin{array}{l}\text { Asymmetric quench } \\
(\sim 0.5 \mathrm{~s})\end{array}$ & $\begin{array}{l}\text { Post quench } 1(\sim 5-10 \\
\text { min) }\end{array}$ \\
\hline Function & $\begin{array}{l}\text { Keep quench interlock } \\
\text { loop closed }\end{array}$ & $\begin{array}{l}\text { Open quench interlock } \\
\text { loop }\end{array}$ & $\begin{array}{l}\text { Keep quench interlock } \\
\text { loop opened }\end{array}$ \\
\hline ID failure mode & OP.1 & AQ.1 & PQ1.1 \\
\hline Failure mode & $\begin{array}{l}\text { Quench interlock loop } \\
\text { opened } 1002 \text { or } 2002\end{array}$ & $\begin{array}{l}\text { Quench interlock loop } \\
\text { not opened } 1002\end{array}$ & $\begin{array}{l}\text { QL is closed locally } \\
1002 \text { or } 2002\end{array}$ \\
\hline Immediate effect & $\begin{array}{l}\text { False energy } \\
\text { extraction, no firing of } \\
\text { the quench heaters, } \\
\text { false circuit quench } \\
\text { interlock }\end{array}$ & $\begin{array}{l}\text { Energy is extracted, } \\
\text { both quench heater } \\
\text { series are fired, circuit } \\
\text { quench interlock is } \\
\text { sent }\end{array}$ & $\begin{array}{l}\text { No effect in this state, if } \\
\text { undetected, higher } \\
\text { probability of missing } \\
\text { interlock in case of } \\
\text { quench }\end{array}$ \\
\hline End effect & False beam dump & Injection delayed (3 h) & Injection delayed $(3 \mathrm{~h})$ \\
\hline Severity of EE & 2 & 2 & 2 \\
\hline Detection Method & $\begin{array}{l}\text { Quench interlock loop } \\
\text { monitoring indicates } \\
\text { loop status }\end{array}$ & $\begin{array}{l}\text { Detector post mortem, } \\
\text { (no final detection if } \\
\text { only loop relay is } \\
\text { broken }\end{array}$ & $\begin{array}{l}\text { Post mortem (loop } \\
\text { falgs, loop open or } \\
\text { closed) }\end{array}$ \\
\hline Notes & $\begin{array}{l}\text { after upgrade } \\
\text { additional loop voltage } \\
\text { monitors (eases fault } \\
\text { localization) }\end{array}$ & $\begin{array}{l}\text { Quench interlock loop } \\
\text { is opened by second, } \\
\text { redundant quench } \\
\text { detector }\end{array}$ & \\
\hline Recommendations & & $\begin{array}{l}\text { put secondary relay } \\
\text { contact in detector }\end{array}$ & \\
\hline
\end{tabular}

Table 1 shows the FMEA table for the output quench interlock loop for three states. The identified end effects, caused by the quench detection system, concern the quadrupole and beam operation:

- QDS_EE1: False quenching, severity level 2 (S2)

- QDS_EE2: Quadrupole damaged, S5 
- QDS_EE3: Injection delayed, S2

- QDS_EE4: False beam dump, S2

- QDS_EE5: Missed beam dump by quench detection system, beam dump by another protection system, $\mathrm{S} 3$

- QDS_EE6: Missed beam dump, S5

Table 2: LHC severity table

\begin{tabular}{|l|l|l|}
\hline \multicolumn{2}{|l|}{ Severity level } & Recovery time \\
\hline 7 & Catastrophic: & $\begin{array}{l}\text { Infinite } \\
\text { Year } \\
\text { Month }\end{array}$ \\
5 & $\begin{array}{l}\text { Very critical: } \\
\text { Critical: } \\
\text { Unsafe/ accident }\end{array}$ & Week \\
\hline 4 & $\begin{array}{l}\text { Major: } \\
\text { Degraded unacceptable /I }\end{array}$ & Day \\
\hline 3 & $\begin{array}{l}\text { Moderate: } \\
\text { Degraded unacceptable / }\end{array}$ & Hours \\
\hline 2 & $\begin{array}{l}\text { Low: } \\
\text { Degraded acceptable }\end{array}$ & Minutes \\
\hline 1 & $\begin{array}{l}\text { Negligible: } \\
\text { Availability }\end{array}$ & \\
\hline
\end{tabular}

\subsection{Step 3: Reliability Targets for Failure Effects}

In this step, the accelerator targets (defined in step 1) are allocated to the end effects (defined in step 2). The failure effects are assigned, depending on their severity category, to the appropriate column in the risk matrix. Then, the column specific accelerator targets are allocated to the quench detection system and then to the failure effects. The allocation is based on experience and involves the consideration of all the accelerator systems, their failure behavior, complexity and achievable reliability. The reliability target of end effect 5 is for example: the unreliability of ten years must be less than $10 \%$.

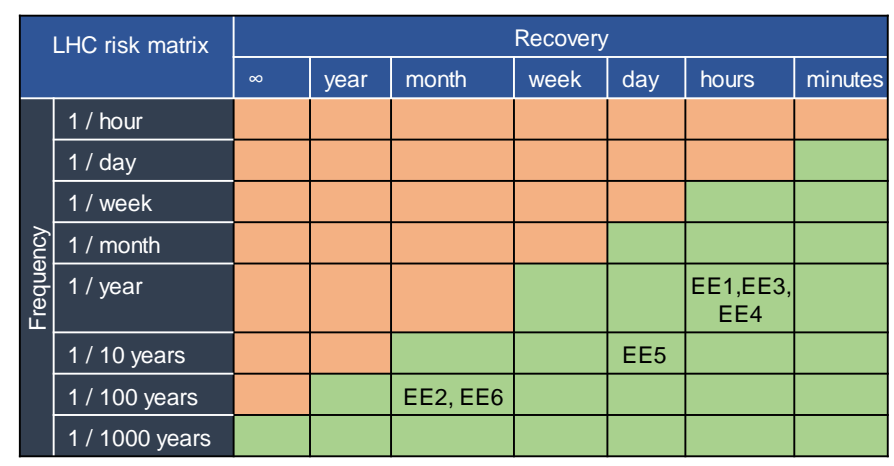

Figure 9: Reliability targets for the quench detection system

\subsection{Step 4: Risk Evaluation}

At this stage, the first task of a reliability analysis, the definition of the reliability goals, is achieved. Furthermore, through failure analysis with FMEA, a comprehensive knowledge of the failure characteristics of the system and the impact on accelerator operation is obtained.

With risk evaluation, the necessary extent of reliability actions is estimated. Risk is evaluated with two criteria: severity and detectability. Each failure effect, classified from S3 (day) to S7 (infinite), has a significant effect on the operation of the accelerator. Failure modes, which are undetectable, independent of the severity category, have the possibility to accumulate and to cause failures of a higher category. Both these failure modes require further studies.

The main information of the FMEA table (Table 1) can be visualized with a fault tree (Figure 10). The top event corresponds to LHC failures. One level lower, the severity categories are distinguished. On the next level are the systems, e.g. the quench detection system. The end effects and failure modes of the corresponding severity category are attached to each quench detection gate. The fault tree has eight gates for end effects, because combinations of the six identified end effects (chapter 3.2.5) exist.

The fault tree model developed with RIRE (Figure 10) provides a bridge to quantitative reliability modeling, where reliability is demonstrated and design solutions are compared. With the risk evaluation it is determined which parts of the fault tree need to be further developed following a deductive approach.

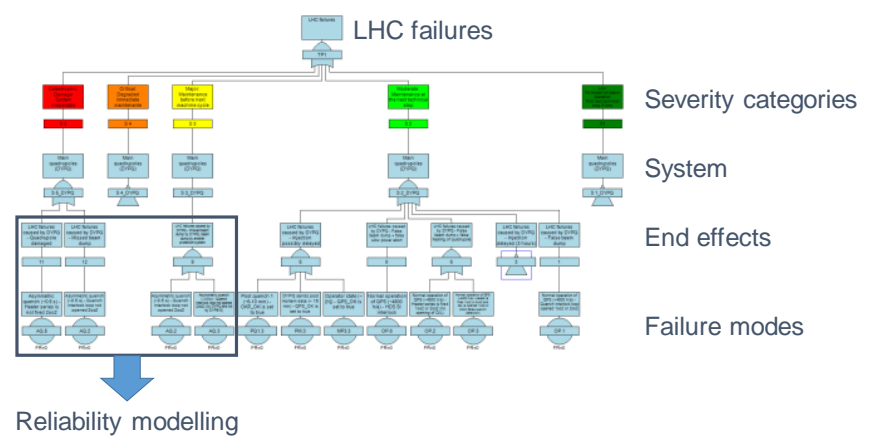

\section{Figure 10: Fault tree visualization [14] of the FEMA table}

Concerning the quench detection system, five end effects are in the severity category two, one end effect is in category 3 and two end effects are in category five. According to the risk evaluation, the end effects of category three and five have to be studied in more detail.

\section{EXAMPLE SPECIFIC CONCLUSIONS AND DISCUSSION}

The reliability modeling of the quench detection system, related to a system upgrade foreseen in 2020, is ongoing. The system failure modes, which are root contributors in the requirements fault tree, become gates and their causes are identified. The present abstraction level varies, depending on the severity category and the available failure rate data. The failure rates are estimated based on experience, failure statistics of similar systems or on prediction standards. The trigger link, as a contributor for the failure mode "two out of two quench heaters are not fired", which has severity category of 5 and the failure effect "quadrupole damaged" is being analyzed. Preliminary results show that the probability, that for one out of 392 quadrupoles no (0oo2) heater is fired within 100 years is $0.1 \%$, which is conform to the initial requirements set in the matrix of the reliability targets.

In addition to finalizing the modeling of the remaining 
critical failure modes, the fault tree will be enlarged sideward, adding other machine protection systems.

Allocating the accelerator reliability requirements to the systems is based on experience by consideration of other accelerator systems, their failure behavior and complexity. A systematic approach for reliability allocation, as for example based on complexity, is needed by which a consistent and reasonable allocation is ensured [].

A next step in the development of the RIRE method would be the application to other large systems, not related to particle accelerators, showing the universal applicability of the described method.

\section{REFERENCES}

1. A. Apollonio, Machine Protection: Availability for Particle Accelerators, PhD thesis, CERN, 2015

2. Some LHC Milestones, CERN bulletin 38/2008, Mon 08 Sep 2008

3. CERN, LHC design report. Vol. 1 the LHC main ring, 2004

4. R. Schmidt et al, Protection of the CERN Large Hadron Collider, in: new journal of physics, 8, p. 290, 2006

5. 2006/42/EC, Directive 2006/42/EC of the European parliament and of the council of 17 May 2006 on machinery, and amending directive 95/16/EC (recast)

6. IEC 61508, Functional safety of electrical/ electronic/ programmable electronic safety related systems, 2010

7. ISO/TR 14121-2:2012, Safety of machinery - Risk assessment - Part 2: Practical guidance and examples of methods, 2013

8. IEC 60812, Analysis techniques for system reliability Procedure for failure mode and effects analysis (FMEA), edition 2.0, 2006

9. IEC 61025, fault tree analysis (FTA), edition 2.0, 2006

10. VDI2221, Systematic approach to the development and design of technical systems and products, 1993

11. ISO/IEC Guide 51:1999, Safety aspects - Guidelines for their inclusion in standards, 3.2

12. UK Health and safety executive, reducing risks, protecting people, HSE books, 2001

13. D. Harel, Statecharts: a visual approach to complex systems, Department of Applied Mathematics, The Weizmann Institute of Science, 1984

14. Isograph Ltd, Reliability Workbench (x64) incorporating Fault Tree+ (13.0.1.0), 2016

15. O. Rey Orozco et al., Availability allocation to particle accelerators by complexity criteria, IPAC 2018, Vancouver, BC, Canada

\section{BIOGRAPHIES}

Miriam Blumenschein, MSc.

Machine Protection and Electrical Integrity Group

European Organization for Nuclear Research CERN

CH-1211 Geneva 23, Switzerland

e-mail: Miriam.Blumenschein@ cern.ch

Miriam Blumenschein graduated from the University of
Stuttgart in 2015 with a MSc. in mechanical engineering. Since 2015 she has been working as a research fellow at the European Organization for Nuclear Research CERN in Geneva, Switzerland. As member of the reliability and availability working group she evaluates the reliability of accelerator protection systems. She is also a member of the Machine Protection and Electrical Integrity group, responsible for the protection of the large scale super conducting magnets at CERN.

Jelena Spasic, PhD.

Machine Protection and Electrical Integrity Group

European Organization for Nuclear Research CERN

CH-1211 Geneva 23, Switzerland

e-mail: Jelena.Spasic@ cern.ch

Jelena Spasic received the BSc. and MSc. degrees in electronics engineering from the Faculty of Electrical Engineering, Belgrade, Serbia, and the PhD degree in computer science from Leiden University, the Netherlands, in 2008 and 2017. From 2008 to 2011, she was an embedded systems researcher and engineer in the Institute Mihailo Pupin, Belgrade, Serbia. Currently, she is an electronics engineer at CERN, developing electronics for protection of magnet circuits in the LHC. Her research interest includes real-time scheduling, performance and energy modeling and transformations in embedded streaming systems, and electronics design techniques for radiation-tolerant systems.

Jens Steckert, MSc.

Machine Protection and Electrical Integrity Group

European Organization for Nuclear Research CERN

CH-1211 Geneva 23, Switzerland

e-mail: Jens.Steckert@ cern.ch

Jens Steckert has been working as engineer in the Machine Protection and Electrical Integrity Group in the Technology Department at CERN since 2008. He earned his MSc. degree in Sensor Systems Technology at the University of Applied Sciences in Karlsruhe, Germany in 2007. His main field is system design, implementation and operation of radiation tolerant electronics for the quench detection system of LHC's superconducting magnets.

Jan Uythoven, PhD.

Machine Protection and Electrical Integrity Group

European Organization for Nuclear Research CERN

CH-1211 Geneva 23, Switzerland

e-mail: Jan.Uythoven@ cern.ch

Jan Uythoven graduated in Physics from Eindhoven University (the Netherlands), after which he obtained a $\mathrm{PhD}$. at Oxford University in 1991. After working in industry, he joined CERN in 1992, where he occupied many different positions. Presently he is a Senior Physicist in charge of the section providing machine safety equipment throughout the accelerator chain at CERN. He is also in charge of the Machine Development programme of the LHC. 